\title{
Austin Powers bites back: a cross sectional comparison of US and English national oral health surveys
}

\author{
@) (1) $\Theta$ OPEN ACCESS
}

\section{Carol C Guarnizo-Herreño research assistant ${ }^{12}$, Georgios Tsakos reader ${ }^{1}$, Aubrey Sheiham emeritus professor (deceased) ${ }^{1}$, Michael G Marmot professor ${ }^{1}$, Ichiro Kawachi professor ${ }^{3}$, Richard G Watt professor $^{1}$}

'Department of Epidemiology and Public Health, University College London, London WC1E 7HB, UK; ${ }^{2}$ Departamento de Salud Colectiva, Facultad de Odontología, Universidad Nacional de Colombia, Bogotá, Colombia; ${ }^{3}$ Department of Social and Behavioral Sciences, Harvard School of Public Health, Boston, Massachusetts 02115, USA

\begin{abstract}
Objective To compare oral health in the US and England and to assess levels of educational and income related oral health inequalities between both countries.

Design Cross sectional analysis of US and English national surveys. Setting Non-institutionalised adults living in their own homes.

Participants Oral health measures and socioeconomic indicators were assessed in nationally representative samples: the Adult Dental Health Survey 2009 for England, and the US National Health and Nutrition Examination Survey 2005-08. Adults aged $\geq 25$ years were included in analyses with samples of 8719 (England) and 9786 (US) for analyses by education, and 7184 (England) and 9094 (US) for analyses by income.

Main outcome measures Number of missing teeth, self rated oral health, and oral impacts on daily life were outcomes. Educational attainment and household income were used as socioeconomic indicators. Age standardised estimates of oral health were compared between countries and across educational and income groups. Regression models were fitted, and relative and absolute inequalities were measured using the relative index of inequality (RII) and the slope index of inequality (SII).
\end{abstract}

Results The mean number of missing teeth was significantly higher in the US (7.31 (standard error 0.15)) than in England (6.97 (0.09)), while oral impacts were higher in England. There was evidence of significant social gradients in oral health in both countries, although differences in oral health by socioeconomic position varied according to the oral health measure used. Consistently higher RII and SII values were found in the US than in England, particularly for self rated oral health. RII estimates for self rated oral health by education were 3.67 (95\% confidence interval 3.23 to 4.17 ) in the US and 1.83 (1.59 to 2.11) in England. In turn, SII values were 42.55 (38.14 to 46.96 ) in the US and 18.43 (14.01 to 22.85 ) in England.

Conclusions The oral health of US citizens is not better than the English, and there are consistently wider educational and income oral health inequalities in the US compared with England.

\section{Introduction}

There is a longstanding belief in the United States that the British have terrible teeth, much worse than US citizens. This view dates back at least 100 years, with toothpaste adverts extolling the virtues of American smiles. ${ }^{1}$ Contemporary examples of this belief in popular US culture range from The Simpsons ${ }^{2}$ to the Hollywood character Austin Powers and his repugnant smile. ${ }^{3}$ The perceived terrible state of British teeth is also perpetuated in contemporary literature. For example, Everett, the annoying and dull English love rival in Donna 
Tartt's Pulitzer Prize winning novel The Goldfinch, is disparagingly noted for his "rabbit teeth."

Few studies have directly compared the oral health of US and UK populations. Two descriptive studies have indicated that US adults had better oral health than their British counterparts. ${ }^{56}$ However, these studies did not statistically assess these differences.

The US and UK share similar political systems and are noted for their high levels of inequality. ${ }^{7}$ One notable policy difference between the countries is the funding and delivery of health care. In the UK dentistry is largely provided through the NHS, whereas in the US dental insurance coverage is dominant. Evidence on differences in health inequalities between these countries is mixed. Some studies showed higher absolute levels of inequality in the US, ${ }^{89}$ whereas others that measured relative inequalities did not find significant differences. ${ }^{10}{ }^{11}$ No study has assessed levels of oral heath inequalities between the US and UK. The aim of this study was to compare the oral health and assess levels of oral health inequalities between the US and England.

\section{Methods \\ Data}

Our analysis was based on data from the English Adult Dental Health Survey (ADHS) and the US National Health and Nutrition Examination Survey (NHANES). Both are nationally representative surveys with comparable information about oral health and socioeconomic position.

In the ADHS 2009, ${ }^{12}$ eligible adults were invited to an interview and those with at least one natural tooth were also invited to a clinical examination. In England, interview data referred to 9663 adults, of whom 5622 completed the clinical examination. The NHANES 2005-06 and 2007-08 collected information on oral health from 11791 adults. $^{13}$

We selected participants aged 25 years and older as many younger people are still studying, and so final educational attainment cannot be known. Analyses were conducted separately for educational and income inequalities, and only for adults with complete data. Information on missing data is included in appendix 1 of the online data supplement. The analytical samples were 8719 (England) and 9786 (US) for analyses by education, and 7184 (England) and 9094 (US) for analyses by income. For clinical oral health, we considered only data for dentate participants in the US to achieve comparability with the English data, as only dentate individuals underwent the ADHS clinical examination. The samples for clinical data were 5048 (England) and 7718 (US) for analyses by education, and 4408 (England) and 7234 (US) for analyses by income.

\section{Variables}

Number of missing teeth, self rated oral health, and oral impacts on daily life were our outcomes. Number of missing teeth was derived from the clinical examination. For self rated oral health, we derived a binary variable distinguishing individuals who perceived their oral health as good or better from those who did not. For oral impacts, both surveys included six identical questions from the Oral Health Impact Profile-14 (OHIP-14) assessing pain, function, and social impacts. In line with standard practice ${ }^{14}{ }^{15}$ we derived a binary measure separating the rest of the sample from those who reported "very often" or "fairly often" to any OHIP-14 question. Although edentulousness (no natural teeth) was self reported in the ADHS 2009 and clinically examined in NHANES 2005-08, we present relevant results as supplementary information (appendix 2).

Educational attainment and household income were the socioeconomic position indicators. Education was categorised as: high (college degree or above), medium (US high school diploma, some qualifications but not college degree in England), and low (less than high school in US, no qualifications in England). Regarding household income, US income data was available as categorical rather than in absolute numbers; we therefore divided the variable in three hierarchical groups approximating to tertiles. To make comparable the income data in England, we used the same categorisation. Age, sex, marital status, and ethnicity were covariates given their relationship with oral health and socioeconomic position.

\section{Statistical analysis}

First, we estimated age standardised estimates of oral health in each country. Second, regression models were fitted to assess the association between oral health and socioeconomic position. For the binary outcomes, we estimated prevalence ratios using robust Poisson regression models. For the count outcome of number of missing teeth, we report incidence rate ratios estimated using negative binomial regression models.

Third, to measure relative and absolute inequalities, the relative index of inequality (RII) and the slope index of inequality (SII) were estimated (appendix 1). The RII and SII are regression based indices that use all available data and take into account the distribution of the population across all socioeconomic categories. ${ }^{16}{ }^{17}$ The RII can be interpreted as the prevalence ratio, and the SII as the absolute difference in prevalence of the outcome between people at the bottom and those at the top of the socioeconomic position hierarchy. RII values $>1$ and SII values $>0$ indicate inequality and show that the outcome is higher among those with a lower level of education or income. All analyses took into account the complex sampling design and survey weights.

\section{Patient involvement}

There was no direct patient involvement in this study. The datasets analysed did not include names or identity numbers of participants.

\section{Results}

Age standardised estimates showed that, among dentate adults, the mean number of missing teeth was significantly higher in the US (7.31 (standard error 0.15)) than in England (6.97 (0.09)) (table $1 \Downarrow$ ). For subjective measures, oral impacts were more prevalent in England while the prevalence of less than good self rated oral health was not significantly different between the countries (table $1 \Downarrow$ ). In both countries, women reported more oral impacts and had more missing teeth than men, while the opposite was true for self rated oral health. Estimates by age group revealed that missing teeth and edentulousness were higher in the US among those aged 25-64 years, whereas number of missing teeth was higher in England among older adults.

Adults in the highest education or income groups tended to have better oral health in the US, except for number of missing teeth in the top income group. Conversely, those in the lowest socioeconomic position levels tended to be better off in England (table $2 \Downarrow$ ).

There were significant associations between oral health and socioeconomic position and a general pattern of social gradients in both countries. These gradients tended to be steeper in the 
US with the exception of the income gradient in the number of missing teeth (appendix 3).

Figure $1 \Downarrow$ shows relative socioeconomic inequalities in oral health. The relative index of inequality (RII) was $>1$ for all outcomes in both countries, indicating that negative outcomes were higher among those with less education and income. Since higher scores in the RII indicate larger inequalities, our results show that relative inequalities tended to be higher in the US. The difference between the two countries was particularly marked for self rated oral health and lower for number of missing teeth. Estimates of absolute inequalities are presented in table $3 \Downarrow$. Absolute educational and income inequalities were consistently higher in the US, with larger differences observed for self rated oral health.

\section{Discussion}

Contrary to popular belief, our study showed that the oral health of US citizens is not better than the English. Indeed, our study showed a mixed picture, with Americans having significantly more missing teeth, the English reporting more oral impacts, and no differences in self rated oral health between the two countries. Adults in the lowest socioeconomic position tended to have better oral health in England, while those at the top educational or income levels were generally better in the US. This was particularly clear for self rated oral health. Consistent education and income gradients in oral health were found in both countries, with steeper gradients in the US. Relative and absolute measures of oral health inequalities were consistently higher in the US, especially for self rated oral health.

It is difficult to compare our results with other studies as no previous research has analysed epidemiological data to determine differences in oral health and inequalities between the US and England.

In this analysis we were not able to explore in depth potential explanations for our findings. However, it is established that certain ethnic minorities have poorer oral health than the majority white population. ${ }^{18-20}$ Clearly the ethnic composition in US and England are different, and this could explain our results. However, repeating our analyses restricting the samples to the white population revealed similar results (appendix 4). In addition, as inequalities in subjective oral health tend to be very low among edentate adults, ${ }^{21}{ }^{22}$ we conducted additional analysis of the subjective outcomes excluding edentate participants, but again the findings were similar (appendix 4).

Differential levels of access and provision of treatment services between the health systems may have contributed to our findings. For example, it is possible that national differences in wisdom teeth extraction might partially explain our findings on missing teeth. Another possible reason could be the role of oral health risk factors such as sugars consumption and smoking. However, a previous study showed that health behaviours did not explain the differences in levels of inequality between these countries. ${ }^{8}$ Moreover the role of health behaviours as explanation of inequalities is rather limited, ${ }^{23}{ }^{24}$ although this would depend on the specific health outcome and behaviours analysed. Finally, wider societal differences in welfare policies exist, with England having a more comprehensive range of "safety net" policies which may help to reduce oral health inequalities.

This analysis has some limitations. First, because of the poor comparability of clinical outcomes between surveys, our analysis was limited to one clinical measure of oral health status, number of missing teeth, and did not include any aesthetic or orthodontic outcomes. Another limitation is the comparability of subjective measures of oral health, as these are sensitive to cultural differences in reporting. However, self reported health outcomes are considered valid for comparisons between countries ${ }^{25}{ }^{26}$ and are accepted as valid indicators of oral health. ${ }^{21-29}$ It would have been preferable to use NHANES 2009-10, which would have been closer in time to ADHS 2009, but this was not possible because of lack of comparable data.

In conclusion we have shown that the oral health of US citizens is not better than the English, and there are consistently wider educational and income related oral health inequalities in the US compared with England.

Contributors: CCG-H, RGW, and GT co-designed the study. CCG-H and GT performed the statistical analysis. RGW and CCG-H wrote the first draft. GT, AS, MGM, and IK read the draft and provided comments. All co-authors read and approved the final draft.

Competing interest: All authors (with the exception of AS) have completed the ICMJE uniform disclosure form at www.icmje.org/coi_ disclosure.pdf (available on request from the corresponding author) and declare: no support from any organisation for the submitted work; no financial relationships with any organisations that might have an interest in the submitted work in the previous three years; no other relationships or activities that could appear to have influenced the submitted work.

Ethical approval: Not necessary because data obtained from secondary sources.

Transparency: This manuscript is an honest, accurate, and transparent account of the study being reported, and no important aspects of the study have been omitted.

Data sharing: Datasets used in this study are available at www.cdc.gov/ nchs/nhanes.htm (NHANES survey) and https://discover.ukdataservice. ac.uk/ (ADHS survey). In these datasets, data are totally anonymised.

1 Grumsen S. The era of whiter teeth: advertising in American dentistry 1910-1950. J Hist Dent 2009;57: 75-84. 19860287

2 Last exit to Springfield. The Simpsons [television programme]. 20th Century Fox, 1993.

3 The Austin Powers series [films]. New Line Cinema, 1997-2002.

4 Tartt D. The goldfinch. Little, Brown, 2013.

5 Crocombe LA, Mejia GC, Koster CR, Slade GD. Comparison of adult oral health in Australia, the USA, Germany and the UK. Aust Dent J 2009;54: 147-53. doi:10.1111/ 1834-7819.2009.01108.x. 19473157

6 Blinkhorn AS. Comparison of the dental health of adults and children living in NSW with their counterparts in the US and UK. N S W Public Health Bull 2009;20: 52-5. doi:10.1071/ NB08067. 19401069

7 Inequality, instability and mobility in family life: the United Kingdom and the United States. Social change: a Harvard-Manchester initiative (SCHMi), Institute for Social Change, University of Manchester, 2013.

8 Banks J, Marmot M, Oldfield Z, Smith JP. Disease and disadvantage in the United States and in England. JAMA 2006;295: 2037-45. doi:10.1001/jama.295.17.2037 16670412

9 McDonough P, Worts D, Sacker A. Socioeconomic inequalities in health dynamics: a comparison of Britain and the United States. Soc Sci Med 2010;70: 251-60. doi:10.1016/ j.socscimed.2009.10.001. 19857919

10 Avendano M, Glymour MM, Banks J, Mackenbach JP. Health disadvantage in US adults aged 50 to 74 years: a comparison of the health of rich and poor Americans with that of Europeans. Am J Public Health 2009:99:540-8. doi:10.2105/A.JPH.2008.139469. 19150903

11 Martinson ML. Income inequality in health at all ages: a comparison of the United States and England.Am J Public Health 2012;102: 2049-56. doi:10.2105/AJPH.2012. 300929. 22994174

12 O'Sullivan I, Lader D, Beavan-Seymour C, Chenery V, Fuller E, Sadler K. Foundation Report: Adult Dental Health Survey 2009 (Technical information). NHS Information Centre, 2011.

13 Dye BA, Barker LK, Li X, Lewis BG, Beltrán-Aguilar ED. Overview and quality assurance for the oral health component of the National Health and Nutrition Examination Survey (NHANES), 2005-08. J Public Health Dent 2011;71:54-61. doi:10.1111/j.1752-7325.2010. 00202.x 21667544

14 Slade GD, Nuttall N, Sanders AE, Steele JG, Allen PF, Lahti S. Impacts of oral disorders in the United Kingdom and Australia. Br Dent J 2005;198: 489-93, discussion 483. doi: 10.1038/sj.bdj.4812252. 15849587

15 Soe KK, Gelbier S, Robinson PG. Reliability and validity of two oral health related quality of life measures in Myanmar adolescents. Community Dent Health 2004;21: 306-11. 15617416

16 Mackenbach JP, Kunst AE. Measuring the magnitude of socio-economic inequalities in health: an overview of available measures illustrated with two examples from Europe. Soc Sci Med 1997;44: 757-71. doi:10.1016/S0277-9536(96)00073-1 9080560

17 Mackenbach JP, Stirbu I, Roskam A-JREuropean Union Working Group on Socioeconomic Inequalities in Health. Socioeconomic inequalities in health in 22 European countries. $N$ Engl J Med 2008;358: 2468-81. doi:10.1056/NEJMsa0707519 18525043

18 Shelley D, Russell S, Parikh NS, Fahs M. Ethnic disparities in self-reported oral health status and access to care among older adults in NYC. J Urban Health 2011;88: 651-62. doi:10.1007/s11524-011-9555-8 21850607 


\section{What is already known on this topic}

There is a popular US belief, dating back many decades, that the English have terrible oral health, much worse than their US contemporaries

However, few studies have directly compared levels of oral health between the US and England or assessed education and income oral health inequalities between these countries

\section{What this study adds}

This is the first analytical study to compare levels of oral health and oral health inequalities between England and the US The mean number of missing teeth was significantly higher in the US, oral impacts were more prevalent in England, and there were no differences in self rated oral health

Both countries displayed significant relative and absolute educational and income related inequalities in all outcomes analysed, and these inequalities were consistently higher in the US than in England

19 Hwang SS, Smith VC, McCormick MC, Barfield WD. Racial/ethnic disparities in maternal oral health experiences in 10 states, pregnancy risk assessment monitoring system, 2004-2006. Matern Child Health J 2011;15: 722-9. doi:10.1007/s10995-010-0643220652385

20 Huang DL, Park M. Socioeconomic and racial/ethnic oral health disparities among US older adults: oral health quality of life and dentition. J Public Health Dent 2015;75: 85-92. doi:10.1111/jphd.12072. 25234710

21 Tsakos G, Demakakos P, Breeze E, Watt RG. Social gradients in oral health in older adults: findings from the English longitudinal survey of aging. Am J Public Health 2011;101: 1892-9. doi:10.2105/AJPH.2011.300215. 21852627

22 Guarnizo-Herreño CC, Watt RG, Fuller E. Socioeconomic position and subjective ora health: findings for the adult population in England, Wales and Northern Ireland. $B M C$ Public Health 2014;14: 827. doi:10.1186/1471-2458-14-827. 25107286

23 Lantz PM, Lynch JW, House JS. Socioeconomic disparities in health change in a longitudinal study of US adults: the role of health-risk behaviors. Soc Sci Med 2001;53: 29-40. doi:10.1016/S0277-9536(00)00319-1 11380160

24 Sabbah W, Tsakos G, Sheiham A, Watt RG. The role of health-related behaviors in the socioeconomic disparities in oral health. Soc Sci Med 2009;68: 298-303. doi:10.1016/j. socscimed.2008.10.030 19027214

25 Bambra C, Pope D, Swami V. Gender, health inequalities and welfare state regimes: a cross-national study of 13 European countries. J Epidemiol Community Health 2009;63: 38-44. doi:10.1136/jech.2007.070292 18768570
26 Robine JM, Jagger CEuro-REVES Group. Creating a coherent set of indicators to monitor health across Europe: the Euro-REVES 2 project. Eur J Public Health 2003;13(suppl): 6-14. doi:10.1093/eurpub/13.suppl_3.6 14533742

27 Borrell LN, Taylor GW, Borgnakke WS, Woolfolk MW, Nyquist LV. Perception of general and oral health in White and African American adults: assessing the effect of neighborhood socioeconomic conditions. Community Dent Oral Epidemiol 2004;32: 363-73. doi:10.1111/ j.1600-0528.2004.00177.x 15341621

28 Borrell LN, Baquero MC. Self-rated general and oral health in New York City adults: assessing the effect of individual and neighborhood social factors. Community Dent Oral Epidemiol 2011;39: 361-71. doi:10.1111/j.1600-0528.2010.00603.x. 21219373

29 Locker D. Self-esteem and socioeconomic disparities in self-perceived oral health. $J$ Public Health Dent 2009:69: 1-8. doi:10.1111/j.1752-7325.2008.00087 x 18662257

\section{Accepted: 01122015}

Published by the BMJ Publishing Group Limited. For permission to use (where not already granted under a licence) please go to http://group.bmj.com/group/rights-licensing/ permissions

This is an Open Access article distributed in accordance with the Creative Commons Attribution Non Commercial (CC BY-NC 3.0) license, which permits others to distribute, remix, adapt, build upon this work non-commercially, and license their derivative works on different terms, provided the original work is properly cited and the use is non-commercial. See: http://creativecommons.org/licenses/by-nc/3.0/. 


\section{Tables}

\begin{tabular}{|c|c|c|c|}
\hline & England & us & Difference ( $P$ value) \\
\hline \multicolumn{4}{|l|}{ Total } \\
\hline Mean (SE) No of missing teeth & $6.97(0.09)$ & $7.31(0.15)$ & 0.001 \\
\hline Self rated oral health lessthan good & 30.84 (29.50 to 32.18$)$ & 31.37 (29.81 to 32.93$)$ & 0.502 \\
\hline Reporting $\geq 1$ oral impact ${ }^{\star}$ & 15.07 (14.05 to 16.08$)$ & 13.46 (12.39 to 14.52$)$ & 0.017 \\
\hline Edentulousness & $6.18(5.64$ to 6.72$)$ & $7.20(6.23$ to 8.17$)$ & 0.844 \\
\hline \multicolumn{4}{|l|}{ Women } \\
\hline Mean (SE) No of missing teeth & $7.09(0.11)$ & $7.44(0.17)$ & 0.010 \\
\hline Self rated oral health lessthan good & 28.67 (26.99 to 30.35 ) & 30.14 (28.29 to 31.99$)$ & 0.167 \\
\hline Reporting $\geq 1$ oral impact ${ }^{\star}$ & 17.15 (15.77 to 18.52$)$ & 15.70 (13.89 to 17.50$)$ & 0.095 \\
\hline Edentulousness & $6.72(6.02$ to 7.42$)$ & 7.63 (6.61 to 8.65$)$ & 0.081 \\
\hline \multicolumn{4}{|l|}{ Men } \\
\hline Mean (SE) No of missing teeth & $6.85(0.13)$ & $7.20(0.18)$ & 0.024 \\
\hline Self rated oral health lessthan good & 33.12 (31.37 to 34.88$)$ & 32.67 (30.61 to 34.74$)$ & 0.700 \\
\hline Reporting $\geq 1$ oral impact ${ }^{*}$ & 12.89 (11.68 to 14.09$)$ & $11.07(10.03$ to 12.10$)$ & 0.021 \\
\hline Edentulousness & $5.32(4.64$ to 6.00$)$ & 6.65 (5.43 to 7.87 ) & 0.296 \\
\hline \multicolumn{4}{|l|}{ Age $25-44$ years } \\
\hline Mean (SE) No of missing teeth & $3.72(0.08)$ & $4.62(0.10)$ & $<0.001$ \\
\hline Self rated oral health lessthan good & 29.12 (27.07 to 31.16$)$ & 28.96 (26.84 to 31.08 ) & 0.899 \\
\hline Reporting $\geq 1$ oral impact ${ }^{*}$ & 15.13 (13.54 to 16.73$)$ & $13.13(12.16$ to 14.09$)$ & 0.045 \\
\hline Edentulousness & $0.21(0.05$ to 0.38$)$ & $0.99(0.47$ to 1.50$)$ & 0.001 \\
\hline \multicolumn{4}{|l|}{ Age $45-64$ years } \\
\hline Mean (SE) No of missing teeth & $7.13(0.14)$ & $7.79(0.20)$ & $<0.001$ \\
\hline Self rated oral health lessthan good & 34.26 (32.20 to 36.32$)$ & 34.10 (31.61 to 36.59$)$ & 0.905 \\
\hline Reporting $\geq 1$ oral impact ${ }^{\star}$ & 16.52 (15.04 to 17.99$)$ & 14.75 (12.73 to 16.78$)$ & 0.083 \\
\hline Edentulousness & $2.91(2.26$ to 3.55$)$ & 5.67 (4.31 to 7.03$)$ & $<0.001$ \\
\hline \multicolumn{4}{|l|}{ Age $\geq 65$ years } \\
\hline Mean (SE) No of missing teeth & $13.07(0.24)$ & $11.76(0.33)$ & $<0.001$ \\
\hline Self rated oral health lessthan good & 28.29 (26.00 to 30.58$)$ & 31.37 (29.19 to 33.54$)$ & 0.039 \\
\hline Reporting $\geq 1$ oral impact ${ }^{\star}$ & 12.44 (10.85 to 14.03$)$ & $11.87(10.30$ to 13.43$)$ & 0.578 \\
\hline Edentulousness & 23.55 (21.42 to 25.67$)$ & 22.02 (19.13 to 24.92$)$ & 0.215 \\
\hline
\end{tabular}


Table 2| Age standardised estimates of oral health measures by socioeconomic position level in England and the US. Values are prevalence $(95 \% \mathrm{Cl})$ unless stated otherwise

\begin{tabular}{|c|c|c|c|c|c|c|}
\hline & \multicolumn{2}{|c|}{ Mean (SE) No of missing teeth } & \multicolumn{2}{|c|}{ Self rated oral health less than good } & \multicolumn{2}{|c|}{ Reporting $\geq 1$ oral impact ${ }^{\star}$} \\
\hline & England & US & England & US & England & US \\
\hline \multicolumn{7}{|l|}{ Educational level } \\
\hline High & $5.70(0.16)$ & $5.56(0.14)$ & 24.53 (22.41 to 26.65$)$ & 15.49 (13.61 to 17.38$)$ & 10.31 (8.87 to 11.76$)$ & 8.01 (6.61 to 9.41$)$ \\
\hline Medium & $7.06(0.12)$ & $7.51(0.17)$ & 32.01 (30.38 to 33.64$)$ & $32.51(30.85$ to 34.18$)$ & $16.40(15.15$ to 17.64$)$ & $13.62(12.14$ to 15.09$)$ \\
\hline Low & $8.19(0.23)$ & $9.58(0.25)$ & 37.39 (33.77 to 41.02 ) & 52.13 (49.33 to 54.94$)$ & 20.94 (17.68 to 24.19$)$ & 21.15 (19.23 to 23.07$)$ \\
\hline$P$ value for trend & $<0.001$ & $<0.001$ & $<0.001$ & $<0.001$ & $<0.001$ & $<0.001$ \\
\hline \multicolumn{7}{|l|}{ Income level } \\
\hline High & $5.76(0.15)$ & $6.16(0.18)$ & 24.01 (22.09 to 25.92 ) & 19.64 (18.19 to 21.08$)$ & $10.40(8.84$ to 11.96$)$ & 7.28 (5.92 to 8.64$)$ \\
\hline Medium & $6.80(0.15)$ & $7.26(0.15)$ & 29.51 (26.84 to 32.18$)$ & 32.16 (30.22 to 34.10$)$ & 13.96 (11.92 to 16.00$)$ & $14.17(12.40$ to 15.94$)$ \\
\hline Low & $7.83(0.17)$ & $8.76(0.25)$ & 36.36 (33.86 to 38.86$)$ & 46.41 (44.08 to 48.73 ) & 20.75 (18.93 to 22.56 ) & 21.10 (19.16 to 23.04$)$ \\
\hline$P$ value for trend & $<0.001$ & $<0.001$ & $<0.001$ & $<0.001$ & $<0.001$ & $<0.001$ \\
\hline
\end{tabular}

*Oral impacts defined in Methods section. 
Table 3| Absolute inequalities in oral health measures, England and the US

\begin{tabular}{|c|c|c|c|}
\hline & No of missing teeth & Self rated oral health less than good & Reporting $\geq 1$ oral impact ${ }^{*}$ \\
\hline \multicolumn{4}{|c|}{ Mean (SE) No of missing teeth or age standardised prevalence } \\
\hline England & $6.97(0.09)$ & 30.84 & 15.07 \\
\hline US & $7.31(0.15)$ & 31.37 & 13.46 \\
\hline \multicolumn{4}{|c|}{ Slope index of inequality $(95 \% \mathrm{Cl}) \dagger$} \\
\hline \multicolumn{4}{|c|}{ Educational inequalities: } \\
\hline England & 3.66 (3.05 to 4.28$)$ & 18.43 (14.01 to 22.85$)$ & 13.51 (10.08 to 16.95$)$ \\
\hline us & $5.00(4.14$ to 5.86$)$ & 42.55 (38.14 to 46.96$)$ & $16.72(12.90$ to 20.54$)$ \\
\hline \multicolumn{4}{|c|}{ Income related inequalities: } \\
\hline England & $3.12(2.46$ to 3.77$)$ & 18.63 (13.59 to 23.67$)$ & $14.66(10.75$ to 18.57$)$ \\
\hline US & 3.61 (2.77 to 4.44$)$ & 33.57 (27.88 to 39.26$)$ & $18.76(14.14$ to 23.41$)$ \\
\hline
\end{tabular}

*Oral impacts defined in Methods section.

†Because the slope index of inequality (SII) is related to the prevalence of the outcome, we have included age standardised prevalence and mean number of missing teeth together with SII in this table. 


\section{Figure}

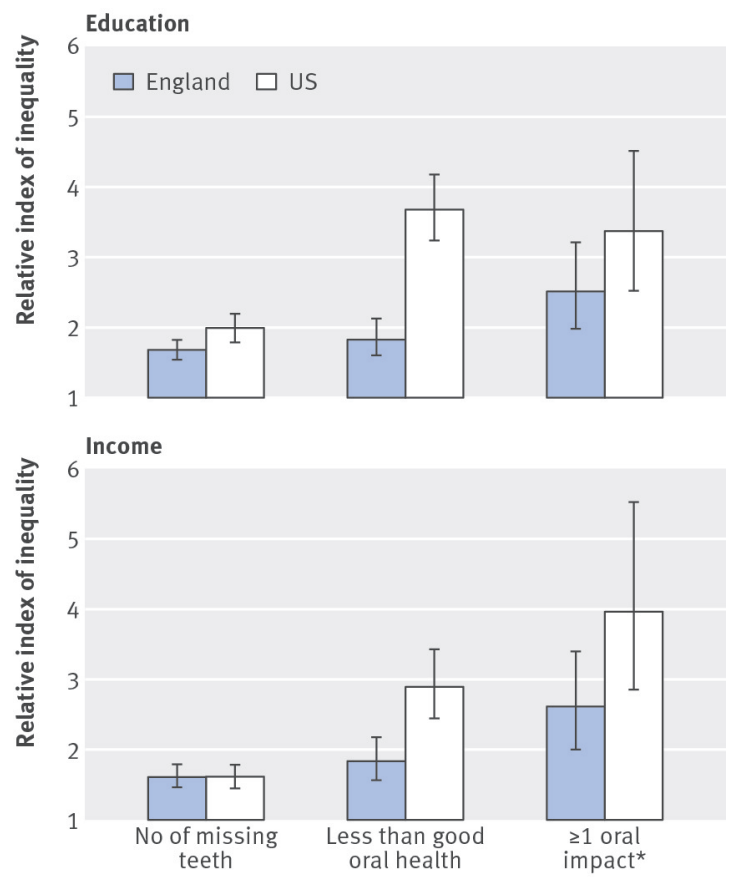

Fig 1 Relative inequalities in oral health measures in England and the US, by education and income 